\title{
Radiological prognostic factors of chronic subdural hematoma recurrence: a systematic review and meta-analysis
}

\author{
Ishita P. Miah ${ }^{1}$ (D) $\cdot$ Yeliz Tank ${ }^{2}$ - Frits R. Rosendaal ${ }^{3} \cdot$ Wilco C. Peul ${ }^{1,4} \cdot$ Ruben Dammers $^{5} \cdot$ Hester F. Lingsma $^{6}$. \\ Heleen M. den Hertog ${ }^{7}$ Korné Jellema ${ }^{4}$. Niels A. van der Gaag ${ }^{4,8}$ on behalf of the Dutch Chronic Subdural \\ Hematoma Research Group
}

Received: 29 July 2020 / Accepted: 16 September 2020 / Published online: 22 October 2020

(C) The Author(s) 2020, corrected publication 2020

\begin{abstract}
Purpose Chronic subdural hematoma (CSDH) is associated with high recurrence rates. Radiographic prognostic factors may identify patients who are prone for recurrence and who might benefit further optimization of therapy. In this meta-analysis, we systematically evaluated pre-operative radiological prognostic factors of recurrence after surgery.

Methods Electronic databases were searched until September 2020 for relevant publications. Studies reporting on CSDH recurrence in symptomatic CSDH patients with only surgical treatment were included. Random or fixed effects meta-analysis was used depending on statistical heterogeneity.

Results Twenty-two studies were identified with a total of 5566 patients (mean age 69 years) with recurrence occurring in 801 patients (14.4\%). Hyperdense components (hyperdense homogeneous and mixed density) were the strongest prognostic factor of recurrence (pooled RR 2.83, 95\% CI 1.69-4.73). Laminar and separated architecture types also revealed higher recurrence rates (RR 1.37, 95\% CI 1.04-1.80 and RR $1.7695 \%$ CI 1.38-2.16, respectively). Hematoma thickness and midline shift above predefined cut-off values $(10 \mathrm{~mm}$ and $20 \mathrm{~mm}$ ) were associated with an increased recurrence rate (RR 1.79, 95\% CI 1.45-2.21 and RR 1.38, 95\% CI 1.11-1.73, respectively). Bilateral CSDH was also associated with an increased recurrence risk (RR 1.34, 95\% CI 0.98-1.84).
\end{abstract}

\section{Key points}

- Recurrence of chronic subdural hematoma (CSDH) after surgery occurs frequently with reported rates that vary between 2.5 and $33 \%$.

- Establishment of radiographic prognostic factors may identify more complex patients prone to CSDH recurrence.

- Many radiological parameters of CSDH have been reported to be associated with the recurrence risk, with conflicting results due to discrepancies in recurrence rate and study heterogeneity.

- In this meta-analysis of 22 studies, we found hyperdense and mixed density hematoma to be associated with the highest risk of CSDH recurrence after surgery, as were laminar and separated hematoma architecture types.

- Awareness of these findings allows for individual risk assessment and might prompt clinicians to tailor treatment measures.

Ishita P. Miah

i.p.miah@lumc.nl; w.c.peul@lumc.nl

Yeliz Tank

y.tank@lumc.nl

Frits R. Rosendaal

f.r.rosendaal@lumc.nl

Wilco C. Peul

w.c.peul@lumc.nl

Ruben Dammers

r.dammers@erasmusmc.nl
Hester F. Lingsma

h.lingsma@erasmusmc.nl

Heleen M. den Hertog

m.h.den.hertog@isala.nl

Korné Jellema

k.jellema@haaglandenmc.nl

Niels A. van der Gaag

n.vandergaag@hagaziekenhuis.nl

Extended author information available on the last page of the article 
Limitations Limitations were no adjustments for confounders and variable data heterogeneity. Clinical factors could also be predictive of recurrence but are beyond the scope of this study.

Conclusions Hyperdense hematoma components were the strongest prognostic factor of recurrence after surgery. Awareness of these findings allows for individual risk assessment and might prompt clinicians to tailor treatment measures.

Keywords Chronic subdural hematoma $\cdot \mathrm{CSDH} \cdot$ Recurrence $\cdot$ Predictors $\cdot \mathrm{CT}$

\section{Introduction}

Chronic subdural hematoma (CSDH) is a frequently encountered neurosurgical disorder of the elderly with a rising incidence $[1,2]$. Historically, CSDH was considered as a progressive and recurrent hemorrhage due to rupture of cortical bridging veins initiated by trauma [3]. Recently however, it has been suggested that a more complex pathway of inflammation, angiogenesis, recurrent micro-hemorrhages, and local coagulopathy in the subdural space is involved [4-8]. This inflammatory response is presumed to play a key role in hematoma formation, re-bleeding, and maintenance.

The diagnosis is based on clinical symptoms and radiological investigation, mostly non-contrast CT scan. Surgery through burr hole drainage or twist drill craniostomy (BHC, TDC) is the mainstay of treatment worldwide $[9,10]$. Alternative strategies include watchful observation or highdose glucocorticoids administration depending on symptom severity and local protocols [11-14]. Ultimately, the aim of all therapeutic modalities is adequate symptom relieve by effective hematoma resolution.

Recurrence of CSDH after surgery occurs frequently with reported rates that vary between 2.5 and 33\% [15-17]. Postoperative closed drainage as interventional measure is effective in reducing recurrence risk by roughly $50 \%[1,10,17]$. Recurrent CSDH poses a formidable challenge in the treatment of symptomatic patients [18]. Recurring symptoms and additional treatment increase patient burden, prolong hospital admissions leading to higher costs, and contribute to a potential poor outcome $[19,20]$. Therefore, the identification of factors associated with recurrence is important for individual risk assessment, treatment decisions, and possibly optimization of pre- and postoperative management. An individualized approach could entail adjusting the timing of surgery and antithrombotic therapy resumption or even exploring alternative treatment strategies depending on local protocols.

Many radiological parameters of CSDH have been reported to be associated with the recurrence risk, including uni- or bilateral hematoma, preoperative hematoma thickness and midline shift, hematoma density and internal architecture, cerebral atrophy, and hematoma volume [21-34]. However, studies have shown conflicting results and large discrepancies in recurrence rates due to heterogeneity in treatment, radiological measurement techniques, and variation in hematoma classifications for hematoma density or architecture.
In this systematic review and meta-analysis, we aimed to identify radiological prognostic factors of CSDH recurrence in surgically treated symptomatic CSDH patients.

\section{Materials and methods}

Before conducting this systematic review, we developed a detailed protocol including objectives and a strategy for collecting and analyzing data. The manuscript was prepared in accordance with the Preferred Reporting Items for Systematic Review and Meta-analysis Protocols (PRISMA) guidelines.

\section{Search strategy and selection criteria}

Literature on symptomatic CSDH patients and radiological findings published until September 2020 were reviewed using PubMed, EMBASE, Web of Science, and Cochrane library. Potential studies were searched using the following keywords and MeSH terms (including abbreviations, variations due to plurality and spelling): "chronic subdural hematoma," "imaging," "radiological," "predictor," "computed tomography," and "magnetic resonance imaging." The search was supplemented by hand searching the reference list of each included article and review article. Our primary outcome was CSDH recurrence. Inclusion criteria for study selection were the following: (1) symptomatic CSDH patients, (2) only surgical therapy by burr hole or twistdrill craniostomy with subdural drainage, (3) pre-defined (and retrievable) definition of CSDH recurrence, (4) follow-up period of $\geq 3$ months, (5) clinical studies with $>10$ subjects, and (6) evaluation of at least one of the following radiological parameters: uni- versus bilateral hematoma, hematoma thickness, midline shift, hematoma density and architecture on CT, hematoma volume, MRI appearance (T1, T2, diffusion-weighted imaging, DWI). Studies performed in animals, case reports or reported in other than English language were excluded.

\section{Data extraction}

Data from the included studies were extracted by one neurologist (IPM) and one radiologist (YT) using a standardized data extraction form. Disagreements were resolved by consensus. The following data were collected: (1) study characteristics (country, study design, year of publication, number of participants, definition of CSDH (diagnostic criteria) and CSDH 
recurrence, type of surgery, follow-up period, radiological parameters evaluated), (2) patient characteristics (mean age, sex, trauma, use of oral anticoagulation, or platelet aggregation inhibitors), and (3) imaging findings of radiological parameters: uni- versus bilateral hematoma, hematoma thickness (frequencies below or above prespecified cutoff value in $\mathrm{mm}$ ), midline shift (present/absent or frequencies below or above prespecified cut-off value in $\mathrm{mm}$ ), hematoma density classification and hematoma architecture types, volume (in $\mathrm{mm}^{3}$, frequencies above or below prespecified cut off value), and MRI appearance (hypo-, iso- or hyperintensity on T1, T2, and DWimaging). Hematoma density was categorized as (1) homogeneous hypodense, (2) homogeneous isodense, (3) homogeneous hyperdense, and (4) mixed density. Hematoma architecture was reported using the four classification as described by Nakaguchi [26] (Table 1, Fig. 1): (a) homogeneous architecture, (b) laminar architecture, (c) separated architecture, and (d) trabecular architecture. Due to heterogeneity and lack of standardization in reporting on hematoma density and architecture, we added two simplified categories to summarize density and architecture findings: (i) a (total) homogeneous group containing all patients with a homogeneous hypodense, homogeneous isodense, and homogeneous hyperdense hematoma; (ii) (total) mixed density group, containing all mixed density hematoma and the following architecture types with mixed density: laminar, separated, grading, and trabecular hematoma.

\section{Quality of reporting in included studies}

We assessed risk of bias and quality of reporting of all included studies based on the Newcastle-Ottawa Quality Assessment Scale (NOS) checklist, used to build a quality score between 0 and a maximum of 9 stars [35]. When there was risk of selection bias in patient inclusion (i.e., exclusion of patients with head trauma, anticoagulant or platelet aggregation inhibitor use, bilateral CSDH, or absence of follow-up CT), one star was subtracted in the selection-section (max. 4 stars). Stars were assigned in the comparability section if adjustments took place for confounders (max. 2 stars). If there was no statement regarding the number of patients who were actually evaluated at the predefined follow up moment (3, 6 , or 12 months), one star was also subtracted in the outcome-section (max. 3 stars). Studies were rated with good quality if they had 3 or 4 stars in the selection domain and 1 or 2 stars in the comparability domain and 2 or 3 stars in the outcome/exposure domain. Studies were of fair quality when they scored 2 stars in the selection domain and 1 or 2 stars in the comparability domain and 2 or 3 stars in the outcome/ exposure domain. Studies were also classified as fair quality when they had maximum stars in the selection and outcome domain, with no stars in the comparability section. Finally, studies were classified as poor quality when they scored 0 or 1 star in the selection domain or 0 stars in comparability domain or 0 or 1 star in outcome/exposure domain.

\section{Statistical analysis}

Analyses were performed using SPSS (version 25.0, IBM Corp) and Review Manager (RevMan, version 5.3. Copenhagen: The Nordic Cochrane Center, The Cochrane Collaboration, 2014). Continuous and categorical variables were summarized with means and counts and percentages respectively. To evaluate recurrence risk, we calculated risk ratios (RR) with 95\% confidence intervals for the following comparisons: (1) unilateral versus bilateral hematoma, (2) hematoma thickness below versus above prespecified cutoff values $(15,20$, and $25 \mathrm{~mm})$, (3) presence versus absence of midline shift, (4) midline shift below versus above prespecified cut off values $(5,10$, and $15 \mathrm{~mm})$, (5) mixed density (total) versus homogenous density (total) hematoma, (6) homogeneous hyper- and mixed density versus homogeneous iso- and hypodensity hematoma, (7) architecture types (homogeneous versus non-homogeneous; laminar versus non-laminar; separated versus non-separated; trabecular versus non-trabecular), (7) hematoma volume below versus above prespecified cut off values (121 $\mathrm{mm}^{3}$ [28]), and (8) hematoma MRI-hypo- and -iso-intensity versus hyper- and mixed intensity on T1, T2, and DW-imaging. Statistical heterogeneity in each
Table 1 Hematoma classification by architecture type

\begin{tabular}{|c|c|}
\hline Architecture types & Description \\
\hline Homogeneous & $\begin{array}{l}\text { Hematoma with complete homogeneous density, including } \\
\text { homogeneous hypo-, iso-, and hyperdense hematoma }\end{array}$ \\
\hline Laminar & $\begin{array}{l}\text { Hematoma with thin high-density layer along the inner } \\
\text { membrane (against the surface of the cortex) }\end{array}$ \\
\hline Separated & $\begin{array}{l}\text { Hematoma with two components of different densities } \\
\text { with a clear boundary between them, resulting in a lower } \\
\text { density component above a higher density component. } \\
\text { If this boundary was mingled at the border, this was } \\
\text { called a gradation type }\end{array}$ \\
\hline Trabecular & $\begin{array}{l}\text { Hematoma with inhomogeneous components and a high } \\
\text { density septum running between the inner and outer } \\
\text { hematoma membrane }\end{array}$ \\
\hline
\end{tabular}


Fig. 1 Hematoma architecture types: a homogeneous; b laminar; c separated; d trabecular type
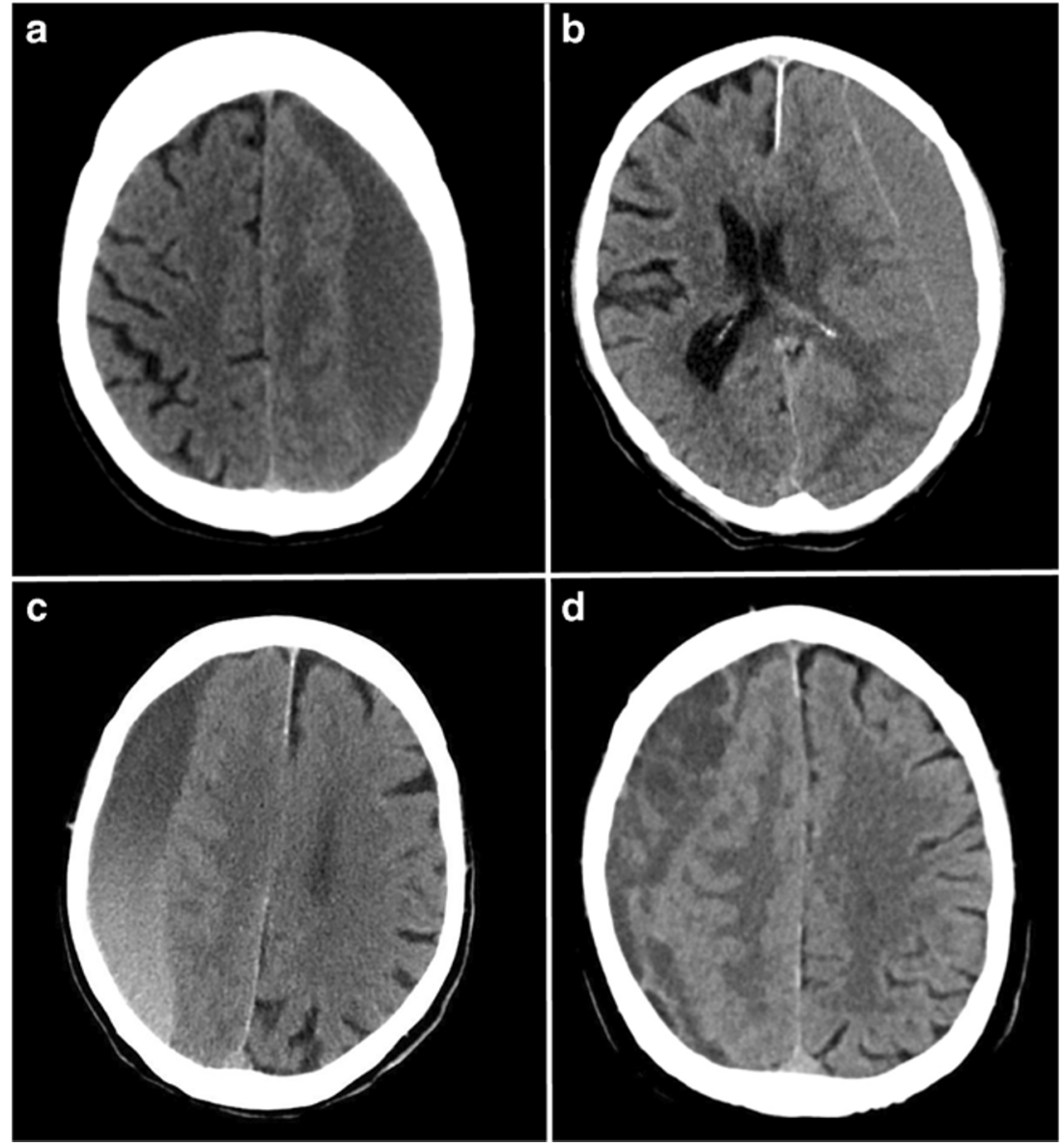

meta-analysis was assessed using the T2, I2, and chi-square tests. When heterogeneity was moderate to high ( $I^{2} 50 \%$ or higher), a random effects model was used; if this was lower than 50\%, a fixed effects model was applied.

\section{Results}

We identified 3112 publications published between 1 January 1940 and September 2020, of which 100 were evaluated in full text and 22 finally included in the meta-analysis (Fig. 2 flow-chart of included studies). All studies scored three to four stars on the selection category of the NOS questionnaire. Scores on the outcome category varied between two to three, depending on the reporting on follow-up. None of the studies adjusted for confounders, resulting in no stars for the comparability section. Study quality was classified as fair for three (14\%) and poor for 19 (86\%) studies (Table 2).

\section{Study and patient characteristics}

All 22 studies were cohort studies, of which three (14\%) had a prospective follow-up design (Table 3 ). Four definitions were identified for CSDH recurrence after primary surgery: (1) surgery (reoperation), without additional clinical or radiological criteria $(n=6)$; (2) clinical symptoms and/or radiological signs requiring additional surgery $(n=1)$; $(3)$ combination of clinical recurrence or progression of symptoms and radiological recurrence or progression of ipsilateral $\mathrm{CSDH}(n=10)$; and finally (4) only radiologic recurrence or progression of CSDH $(n=5)$. In three of these latter five studies, all patients received additional surgery due recurrent or progressive symptoms [29, $45,49]$. One study reported a reoperation in 16 out of 21 cases (76\%) due to reappearance of symptoms with observation only in the remaining patients [26]. The fifth study mentioned a reoperation was performed if reappearance of symptoms accompanied the radiological $\mathrm{CSDH}$ recurrence, without describing the number of patients requiring surgery however [20]. An overview of the radiological parameters evaluated in this meta-analysis is provided in Table 3. Follow-up period ranged from 3 to 12 months. Six patients died prior to discharge [21, 45], leading to a total inclusion of $5566 \mathrm{CSDH}$ patients in the meta-analysis with CSDH recurrence occurring in 801 (14.4\%; Table 4). Overall male-female ratio was 3:1 with a mean age of 68.9 years (SD $4.1 ; n=18$ studies) and a precipitating head trauma in 2089 patients $(62.6 \% ; n=17$ studies). Fourteen hundred and thirty-eight patients had used 
Table 2 Newcastle-Ottawa Quality Assessment Scale (NOS), cohort studies anti-thrombotic agents $(28.9 \% ; n=17$ studies $)$ with the use of anticoagulation in $517(10.4 \%, n=11$ studies $)$, platelet aggregation inhibitors (PAI) in $829(18.1 \%, n=10)$, and unspecified therapy in 92 patients $(2.0 \%, n=5$ studies $)$. All patients were treated by BHC with subdural drainage during 24 to $72 \mathrm{~h}$ (Table 4).

\section{Imaging findings: hematoma laterality, thickness, and midline shift}

Nineteen studies reported on uni- and bilaterality with incomplete data in two $[43,44]$, resulting in seventeen studies with a total of 4400 patients for laterality analysis with a high study heterogeneity $\left(I^{2}=70 \%\right)$. Patients with bilateral $\mathrm{CSDH}$ had higher hematoma recurrence than patients with a unilateral CSDH (Fig. 3a, RR 1.34, 95\% CI 0.98-1.84).

Six studies with a total of 2150 patients reported on hematoma thickness using a cutoff value of $15(n=1), 20(n=4)$, or $25(n=1) \mathrm{mm}$. The largest group comparison showed that the recurrence rate of patients with a $\mathrm{CSDH}$ thickness of more than $20 \mathrm{~mm}$ was higher than patients with a hematoma thickness of less than $20 \mathrm{~mm}$ (Fig. 3b, RR 1.38, 95\% CI 1.111.73). Adding the studies with cut off values of 15 or 25 $\mathrm{mm}$, a similar result was seen (combined group: RR 1.46,
95\% CI 1.19-1.79). Study heterogeneity was low in both comparisons $\left(I^{2}=21 \%\right.$ and $I^{2}=5 \%$ respectively).

Thirteen studies with a total of 2874 patients described midline shift employing a cutoff value of $5(n=1), 10(n=$ $8)$, or $15 \mathrm{~mm}(n=1)$ or reported only on the presence or absence of midline shift $(n=3)$. Patients with a midline shift more than $10 \mathrm{~mm}$ had a higher recurrence rate than patients with a midline shift below $10 \mathrm{~mm}$ (Fig. 3c, RR 1.79, 95\% CI 1.45-2.21). For the combined midline shift groups (adding results of $5 \mathrm{~mm}$ and $15 \mathrm{~mm}$ to $10 \mathrm{~mm}$ ), recurrence risk remained significantly higher (RR 1.76, 95\% CI 1.45-2.14). In the three studies describing only absence or presence of midline shift, there was no difference between the groups (RR 0.82, 95\% CI 0.39-1.72). Study heterogeneity was low in all three comparisons $\left(I^{2}=32 \%, I^{2}=14 \%\right.$, and $I^{2}=0$ respectively).

\section{Imaging findings: hematoma density and architecture}

Seventeen studies with a total of 3813 patients reported on hematoma density. In fifteen studies $(n=3614)$, data were reported or could be reconstructed on homogeneity of the hematoma and mixed density categories. There was a higher risk of recurrence in patients with a mixed density hematoma than in patients with a (complete hypo-, iso-, or hyperdense) homogeneous hematoma (Fig. 4a, RR 1.64, 95\% CI 1.14- 
Fig. 2 Flow-chart of included studies

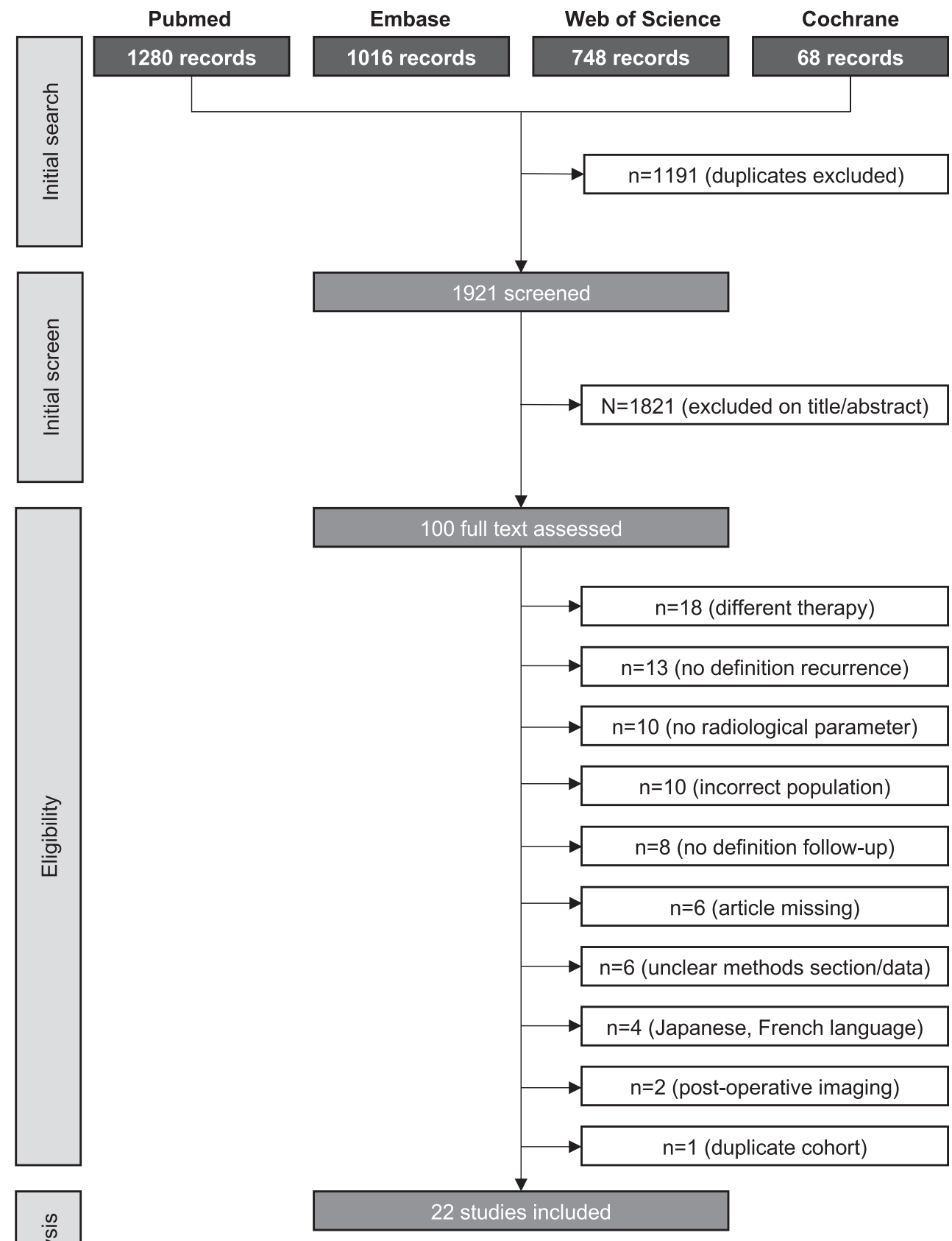

2.37). Eleven studies reported on homogeneous iso- and hypodensity versus hyper- and total mixed density hematomas. Patients with hyper- and mixed density hematomas had more often CSDH recurrence than patients with homogeneous hypo- and isodensity hematomas (Fig. 4b, RR 2.38, 95\% CI 1.69-4.73). Study heterogeneity was high in both comparisons $\left(I^{2}=74 \%\right.$ and $I^{2}=71 \%$ respectively).

Nine studies with a total of $1965 \mathrm{CSDH}$ patients reported on hematoma architecture by evaluation of all four predefined categories. Patients with laminar or separated architecture had a higher risk of hematoma recurrence than those with hematomas in which these features were not present (Fig. 5a, RR 1.37, 95\% CI 1.04-1.80; and Fig. 5b, RR 1.76, CI 95\% 1.382.16 , respectively). Study heterogeneity was low in both comparisons $\left(I^{2}=0\right.$ and $I^{2}=43$ respectively). There was no difference in hematoma recurrence for trabecular architecture (RR 0.88, 95\% CI 0.52-1.49), with high study heterogeneity $\left(I^{2}=61 \%\right)$. 
Table 3 Study characteristics

\begin{tabular}{|c|c|c|c|c|c|c|c|c|}
\hline Study & Country & Period & Design & $\begin{array}{l}\text { Patients } \\
\text { (n) }\end{array}$ & $\begin{array}{l}\text { Definition } \\
\text { CSDH }\end{array}$ & $\begin{array}{l}\text { Definition } \\
\text { reCSDH }\end{array}$ & $\begin{array}{l}\text { Follow-up } \\
\text { (months) }\end{array}$ & $\begin{array}{l}\text { Radiological } \\
\text { parameter }\end{array}$ \\
\hline Won et al. 2020 [36] & Germany & 2016-2018 & Retro. & 389 & No & $\mathrm{S}$ & 3 & $\mathrm{~L}$ \\
\hline Shen et al. 2019 [21] & China & 2012-2018 & Retro. & $461^{\mathrm{a}}$ & No & $\mathrm{C}+\mathrm{R}$ & 3 & $\mathrm{~L}, \mathrm{~T}, \mathrm{M}, \mathrm{D}, \mathrm{A}$ \\
\hline You et al. 2018 [27] & China & 2013-2016 & Retro. & 226 & No & $\mathrm{C}+\mathrm{R}+\mathrm{S}$ & 12 & $\mathrm{~L}, \mathrm{M}, \mathrm{D}, \mathrm{A}$ \\
\hline Yan et al. 2018 [28] & China & $2010-2017$ & Retro. & 231 & No & $\mathrm{C}+\mathrm{R}$ & 3 & $\mathrm{~L}, \mathrm{~T}, \mathrm{M}, \mathrm{D}, \mathrm{A}, \mathrm{V}$ \\
\hline Lee et al. 2018 [44] & Korea & $2012-2015$ & Retro. & 131 & Yes $^{\mathrm{b}}$ & $\mathrm{C}+\mathrm{R}$ & 6 & D, MRI-DWI \\
\hline Bartek et al. 2017 [9] & Sweden & 2005-2010 & Retro. & 759 & No & $\mathrm{S}$ & 6 & $\mathrm{~L}, \mathrm{D}$ \\
\hline Hammer et al. 2017 [43] & Germany & 2009-2012 & Pros. & 73 & No & $\mathrm{S}$ & 1.5 & $\mathrm{D}, \mathrm{A}$ \\
\hline Kim et al. 2017 [45] & Korea & $2010-2015$ & Retro. & $248^{\mathrm{c}}$ & No & $\mathrm{R}$ & 6 & $\mathrm{~L}, \mathrm{D}$ \\
\hline Han et al. 2017 [19] & Korea & 2004-2014 & Retro. & 756 & No & $\mathrm{S}$ & 6 & $\mathrm{~T}, \mathrm{M}$ \\
\hline Stavrinou et al. 2017 [46] & Germany & 2011-2014 & Retro. & 195 & No & $\mathrm{S}$ & 3 & $\mathrm{~L}, \mathrm{D}, \mathrm{A}$ \\
\hline Goto et al. 2015 [47] & Japan & 2004-2010 & Retro. & 414 & No & $\mathrm{C}+\mathrm{R}$ & 6 & $\mathrm{~L}, \mathrm{MRI}-\mathrm{T} 1+\mathrm{T} 2$ \\
\hline Jung et al. 2015 [25] & Korea & 2008-2012 & Retro. & 182 & No & $\mathrm{S}$ & 12 & $\mathrm{~L}, \mathrm{M}, \mathrm{D}, \mathrm{A}$ \\
\hline Song et al. 2014 [29] & Korea & 2009-2012 & Retro. & 97 & Yes $^{\mathrm{b}}$ & $\mathrm{R}$ & 3 & $\mathrm{~L}, \mathrm{~T}, \mathrm{M}, \mathrm{D}$ \\
\hline Jeong et al. 2014 [32] & Korea & 2008-2012 & Retro. & 125 & No & $\mathrm{C}+\mathrm{R}$ & 3 & L, M, D \\
\hline Huang et al. 2014 [30] & Taiwan & $2005-2006$ & Retro. & 94 & Yes $^{\mathrm{d}}$ & $\mathrm{C}+\mathrm{R}$ & 3 & $\mathrm{~L}, \mathrm{M}$ \\
\hline Stanisic et al. 2013 [48] & Norway & 2008 & Pros. & 107 & Yes $^{\mathrm{b}}$ & $\mathrm{C}+\mathrm{R}$ & 7 & $\mathrm{~L}, \mathrm{M}, \mathrm{D}, \mathrm{A}$ \\
\hline Chon et al. 2012 [23] & Korea & 2006-2011 & Retro. & 420 & No & $\mathrm{C}+\mathrm{R}$ & 3 & $\mathrm{~L}, \mathrm{~T}, \mathrm{M}, \mathrm{D}, \mathrm{A}$ \\
\hline Ko et al. 2008 [24] & Korea & 2001-2006 & Retro. & 255 & Yes $^{\mathrm{b}}$ & $\mathrm{C}+\mathrm{R}$ & 3 & $\mathrm{~L}, \mathrm{M}, \mathrm{D}$ \\
\hline Amirjamshidi et al. 2007 [20] & Iran & $2000-2006$ & Pros. & 82 & No & $\mathrm{R}$ & 3 & $\mathrm{~T}, \mathrm{M}, \mathrm{D}$ \\
\hline Yamamoto et al. 2003 [49] & Japan & $1991-2000$ & Retro. & 105 & No & $\mathrm{R}$ & 3 & $\mathrm{~L}, \mathrm{M}$ \\
\hline Nakaguchi et al. 2001 [26] & Japan & 1989-1998 & Retro. & 106 & Yes $^{\mathrm{b}}$ & $\mathrm{R}$ & 3 & $\mathrm{D}, \mathrm{A}$ \\
\hline Oishi et al. 2001 [50] & Japan & 1995-1999 & Retro. & 116 & No & $\mathrm{C}+\mathrm{R}$ & 3 & L, D \\
\hline
\end{tabular}

$A$ architecture; $B H C+D$ burr hole craniostomy combined with post-operative subdural closed drainage system, $C$ clinical recurrence/progression of symptoms, $D$ density, $L$ laterality, $M$ midline shift, Pros prospective, $R$ radiologic recurrence/progression of CSDH, Retro retrospective, $S$ surgery, $T$ thickness

${ }^{a}$ Four patients died before discharge, therefor analyses were performed in 457 patients

${ }^{\mathrm{b}}$ Definition CSDH: radiologic finding of subdural fluid collection with peri-operative confirmation of CSDH

${ }^{\mathrm{c}}$ Two patients died before discharge, therefor analyses were performed in 246 patients

${ }^{\mathrm{d}}$ Definition CSDH: Diagnosis is based on pre-defined radiologic criteria with peri-operative confirmation of CSDH

\section{Imaging findings: hematoma volume and MRI- sequences}

One study $(n=514)$ reported on hematoma volume with frequencies above or below a prespecified cut off value of 121 $\mathrm{mm}^{3}$ based on the receiver operating characteristics (ROC) curve, with the highest recurrence rates in hematomas with a baseline volume above $121 \mathrm{~mm}^{3}$ [28].

Two studies described results on MRI-sequences in relation to CSDH recurrence. The first study $(n=414)$ reported data on the predictive value of MRI-T1 and -T2 sequences, revealing the $\mathrm{T} 1$ classification to be the only prognostic predictor for CSDH recurrence in T1-iso/hypo-intensity group relative to the T1-hyperintensity group [47]. The second study $(n=131)$ revealed more CSDH recurrence when baseline MRI showed DWI hyperintensity compared to hypointensity [44].

\section{Discussion}

In this meta-analysis including over 5500 patients, we identified prognostic factors on CT for recurrence of surgically treated CSDH patients. Hyperdense and mixed density hematoma were associated with the highest risk of CSDH recurrence, as were laminar and separated architecture hematomas. In addition, CSDH with greater magnitude of hematoma thickness and midlines shift carried an increased risk for recurrence.

The establishment of radiological prognostic factors for $\mathrm{CSDH}$ recurrence is of importance in the identification of vulnerable symptomatic CSDH patients for poor outcome and retreatment $[19,20]$. This population would benefit most from optimization of therapy. Many preoperative radiological parameters have been reported as prognostic factors for CSDH recurrence, but results are conflicting [21-34, 51]. 
Table 4 Patient characteristics

\begin{tabular}{|c|c|c|c|c|c|c|c|c|}
\hline Study & Patients $(n)$ & Gender (M:F) & Age (year) & Trauma $(n, \%)$ & $\mathrm{OAC}(n, \%)$ & $\operatorname{PAI}(n, \%)$ & $\begin{array}{l}\text { OAC or PAI } \\
(n, \%)\end{array}$ & $\begin{array}{l}\text { reCSDH } \\
(n, \%)\end{array}$ \\
\hline Won et al. 2020 [36] & 389 & $250: 139$ & - & $202(52)$ & 183 & - & - & $104(27)$ \\
\hline Shen et al. 2019 [21] & $461^{\mathrm{a}}$ & $376: 81$ & 69 & $235(51)$ & - & - & $28(6)$ & $69(15)$ \\
\hline You et al. 2018 [27] & 226 & $184: 42$ & 65 & $161(71)$ & - & - & $14(6)$ & $34(15)$ \\
\hline Yan et al. 2018 [28] & 231 & $188: 43$ & - & - & - & - & - & $33(14)$ \\
\hline Lee et al. 2018 [44] & 131 & $85: 46$ & 68 & $71(54)$ & - & - & $35(27)$ & $7(5)$ \\
\hline Bartek et al. 2017 [9] & 759 & $514: 245$ & 74 & - & $116(15)$ & $194(26)$ & $310(41)$ & $85(11)$ \\
\hline Hammer et al. 2017 [43] & 73 & $47: 26$ & 75 & - & - & - & - & $19(26)$ \\
\hline Kim et al. 2017 [45] & $248^{b}$ & $173: 73$ & 69 & $187(75)$ & $6(2)$ & $53(21)$ & $59(24)^{\mathrm{c}}$ & $31(13)$ \\
\hline Han et al. 2017 [19] & 756 & $574: 182$ & 68 & - & $81(11)$ & $220(29)$ & $301(40)$ & $104(14)$ \\
\hline Stavrinou et al. 2017 [46] & 195 & 134:61 & 71 & $99(51)$ & $48(25)$ & $56(29)$ & $104(53)$ & $35(18)$ \\
\hline Goto et al. 2015 [47] & 414 & $279: 135$ & 77 & - & $14(3)$ & $70(17)$ & $84(20)$ & $37(9)$ \\
\hline Jung et al. 2015 [25] & 182 & $131: 51$ & 68 & $125(69)$ & $10(5)$ & $36(20)$ & $46(25)$ & $25(14)$ \\
\hline Song et al. 2014 [29] & 97 & $64: 33$ & 70 & $61(63)$ & - & - & - & $16(16)$ \\
\hline Jeong et al. 2014 [32] & 125 & $92: 33$ & 69 & $81(65)$ & - & $35(28)$ & $35(28)$ & $8(6)$ \\
\hline Huang et al. 2014 [30] & 94 & $79: 15$ & 69 & $70(74)$ & $2(2)$ & $12(13)$ & $14(15)$ & $13(14)$ \\
\hline Stanisic et al. 2013 [48] & 107 & $72: 35$ & 72 & $86(80)$ & $15(14)$ & $36(34)$ & $51(48)$ & $17(16)$ \\
\hline Chon et al. 2012 [23] & 420 & $334: 86$ & 67 & $237(56)$ & $34(8)$ & $117(28)$ & $151(36)$ & $92(22)$ \\
\hline Ko et al. 2008 [24] & 255 & $150: 105$ & 65 & $181(71)$ & $8(3)$ & - & $8(3)$ & $24(9)$ \\
\hline Amirjamshidi et al. 2007 [20] & 82 & $67: 15$ & 59 & $52(63)$ & - & - & - & $10(12)$ \\
\hline Yamamoto et al. 2003 [49] & 105 & $73: 32$ & 71 & $78(74)$ & - & - & $4(4)$ & $11(10)$ \\
\hline Nakaguchi et al. 2001 [26] & 106 & $82: 24$ & 67 & $63(28)$ & - & - & - & $17(16)$ \\
\hline Oishi et al. 2001 [50] & 116 & $84: 32$ & 72 & $100(86)$ & - & - & $11(9)$ & $10(9)$ \\
\hline
\end{tabular}

$O A C$ oral anticoagulation, $P A I$ platelet aggregation inhibitor, $r e C S D H \mathrm{CSDH}$ recurrence

${ }^{a}$ Four patients died before discharge, therefor analyses were performed in 457 patients

${ }^{\mathrm{b}}$ Two patients died before discharge, therefor analyses were performed in 246 patients

"Because of rounding, percentages in combined "OAC or PAI" group may differ with one percent from the sum of "OAC" and "PAI"

Overall, we found homogeneous hyperdense and mixed density hematoma to be associated with increased recurrence rates. Hematoma density relative to brain parenchyma on a CT image represents the proportion of fresh blood, with hypodense areas representing hematoma of older age and hyperdense components of more recent or active bleeding [52-55]. This imaging appearance reflects the protein concentration from plasma exudation with higher concentration in hyperdense hematoma $[26,38,50,56,57]$. In experimental studies, blood evokes an inflammatory reaction in the subdural space $[42,58]$. This inflammatory reaction is associated with a high amount of inflammatory markers and causes the $\mathrm{CSDH}$ to be more active and is presumed to play a part in hematoma persistence, a greater tendency for re-bleeding and recurrence [39, 58-61]. Novel experimental approaches have evaluated pharmacological adaption of endothelial barrier function, modifying endothelial permeability and plasma exudation $[62,63]$. However, reproducible animal models of human CSDH are not established yet [42]. Results of this meta-analysis are in concordance with the abovementioned pathophysiology of protein concentration in the subdural space, which also explains why lower recurrence rates were found in iso- and hypodense CSDH than in hyperdense hematomas.

Besides categories that describe density of the hematoma, internal architecture types are also used for classification. An established and commonly used classification is that of Nakaguchi (homogeneous, laminar, separated, trabecular), corresponding to proposed stages in natural history of a CSDH [26]. Overall, we found a higher recurrence risk in laminar and separated hematoma than in other hematomas. Several individual studies, however, did not report a high recurrence rate in laminar hematoma [29, 43, 64], but did report trabecular hematoma, corresponding to hematoma with multiplicity of cavities, to reoccur more often [49, 65-69]. This variation and discrepancy is most likely caused by the many available architecture categories which are applied parallel to the classification of Nakaguchi (i.e., loculated hematoma, hematoma with multiplicity of cavities, layered type hematoma, organized hematoma, and niveau formation), but 


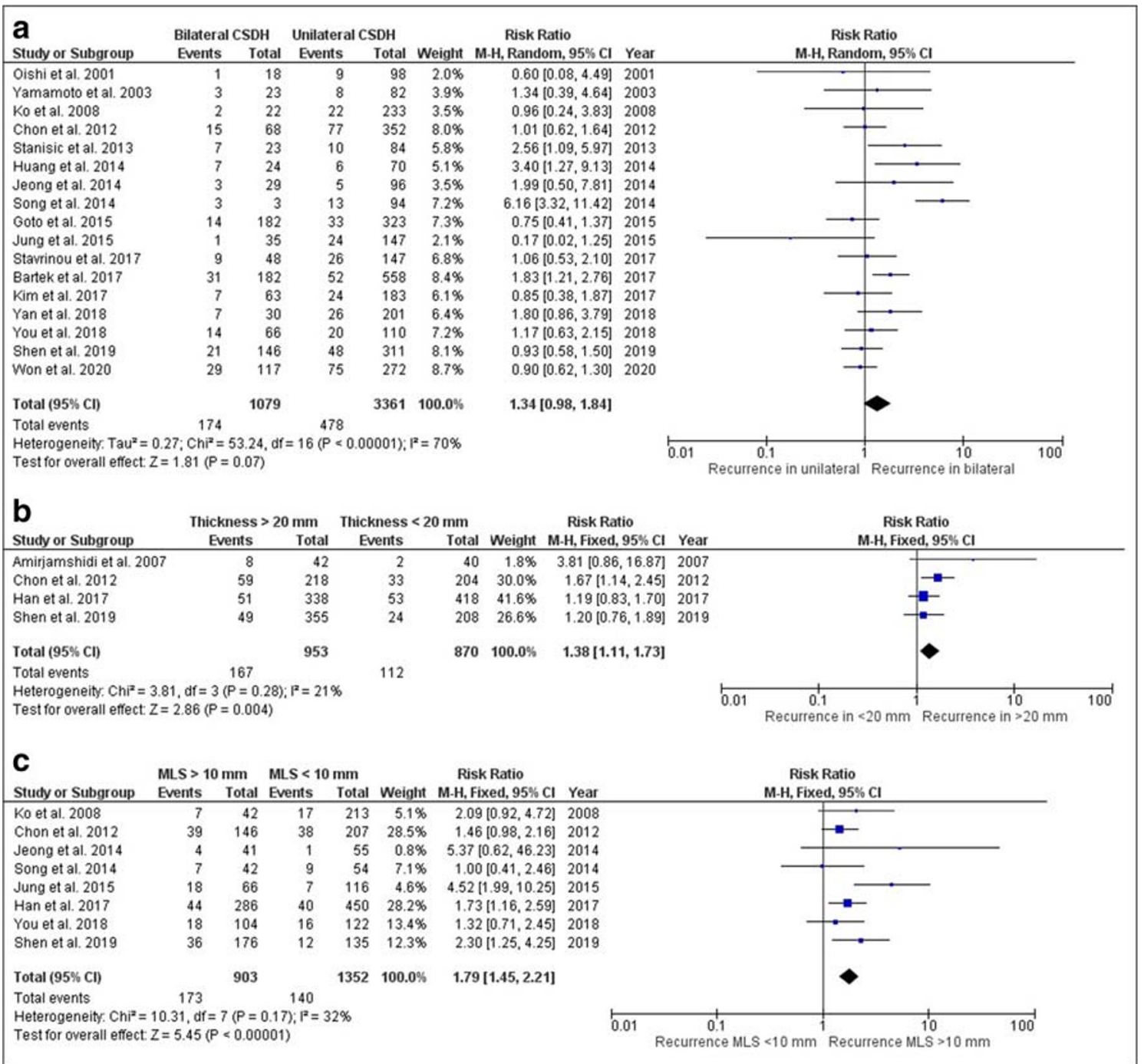

Fig. 3 Forest plot on CSDH recurrence: a uni- versus bilateral hematoma; $\mathbf{b}$ hematoma thickness $<$ or $>20 \mathrm{~mm}$; $\mathbf{c}$ midline shift $<$ or $>10 \mathrm{~mm}$

could also be due to difficulties in applying the classification correctly $[18,26,45,59,70,71]$. In addition, complex internal architecture categories might be very informative, but application can lead to significant intra- and interobserver variability compromising generalizability. In this paper, we propose and demonstrate the benefit of a simplified hematoma classification system based on hematoma density solely. This comprises of a homogeneous iso- and hypodensity category and a second category of CSDH with hyperdense components. This simplified classification could be easy to apply in daily practice with presumably low inter- and intra-observer variation and good insight in the recurrence risk. Future research should confirm the significance of this finding, and also whether adding the different architecture subcategories yields valuable surplus information.

We demonstrate that a greater magnitude of hematoma thickness and a midline shift is associated with an increased recurrence risk. Increased CSDH size and midline shift are often attributed to brain atrophy in close relation to aging, providing the CSDH a potential space to increase easily $[37,40]$. Previous studies have shown cerebral atrophy to be a potential risk factor for CSDH recurrence $[20,50]$. The intracranial (counter-) pressure from brain volume reflects the elasticity of brain parenchyma and may play a part in optimal hematoma absorption [40, 41]. Due to a decrease in brain elasticity and counter pressure by advanced age and atrophy post-operative, reexpansion might potentially be less effective leaving a larger postoperative subdural space that could facilitate persistence or recurrence of CSDH [23, 41, 72]. This mechanism may also explain the increased recurrence in bilateral CSDH. In daily practice, grading of cerebral atrophy is a challenging and difficult task at the time of $\mathrm{CSDH}$ - diagnosis. The compression caused by the subdural hematoma on the involved hemisphere distorts the gyri sulci pattern due to the raised intracranial pressure and complicates a reliable assessment. Furthermore, several scales exist to classify atrophy, causing once again large inter- 


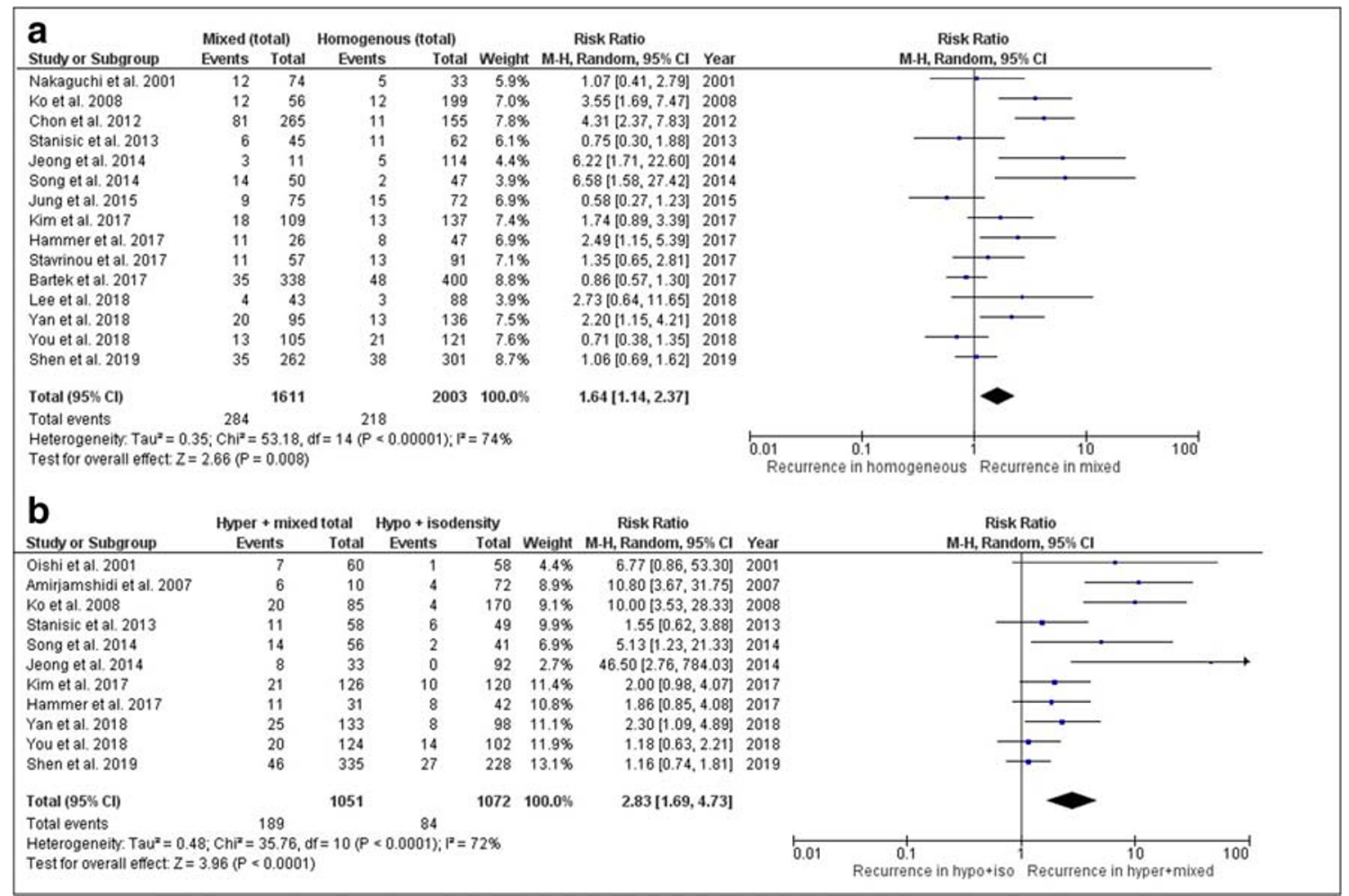

Fig. 4 Forest plot on CSDH recurrence: a homogeneous versus mixed density hematoma; $\mathbf{b}$ iso- and hypodensity versus hyper- and mixed density hematoma

and intra-observer variation. Further evaluation of this parameter was therefore beyond the scope of this meta-analysis.
Recurrence risk is influenced by patient as well as radiological hematoma characteristics. Since a non-contrast

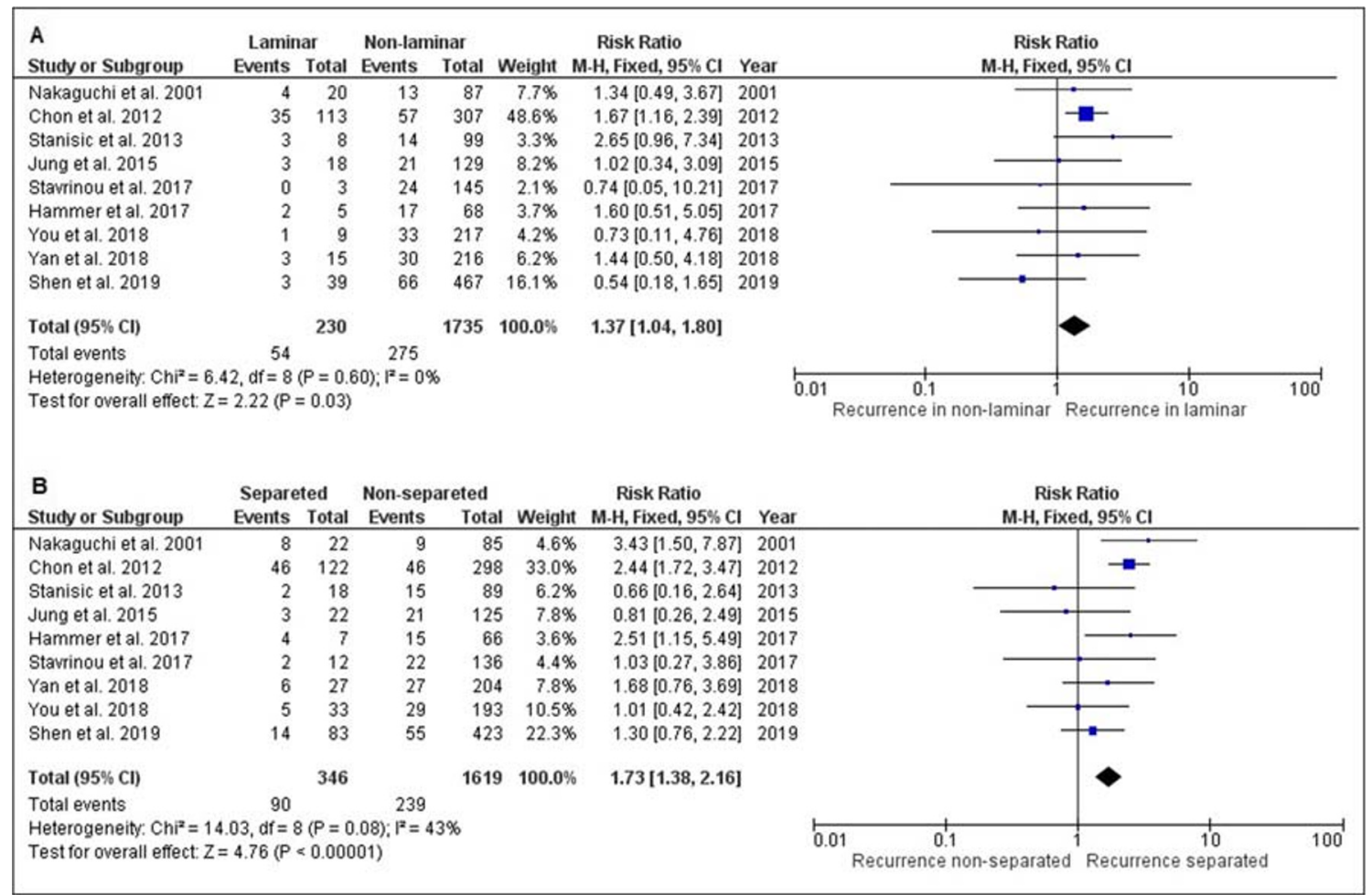

Fig. 5 Forest plot on CSDH recurrence: a laminar hematoma architecture; $\mathbf{b}$ separated hematoma architecture 
CT scan is the most frequently performed diagnostic modality, evaluation of CT predictors is of great additional value next to other presumed clinical predictors such as age, concomitant chronic illness or coagulopathy [21, 23, 28, 64, 71, 73-75]. Similar to the limitations of studies evaluating the value of radiological predictors in recurrence risk, varying results have also been published regarding the effect of age, sex, anticoagulant use, and chronic illness [33, 72, 76, 77]. The addition of radiological predictors of recurrences to baseline patient characteristics for risk calculation may facilitate clinicians to identify patients prone to recurrence more accurately. These findings could lead to adaptation of treatment measures on an individual basis in order to lower the recurrence risk, for example by postponing surgical drainage when hyperdense components are present or adjusting the (local standard) term for anticoagulant therapy resumption post-operative. Limited data also suggest that the addition of corticosteroids might be beneficial in reducing recurrence risk in high-risk patients [73].

Limitations of this meta-analysis are due to methodological aspects of the included studies. We encountered significant heterogeneity in the definitions used for CSDH recurrence, i.e., only radiological recurrence, or the combination of recurring symptoms with radiological persistence or progression of $\mathrm{CSDH}$, or merely re-operation without clarifying the criteria for reoperation. Furthermore, differences in duration of follow-up, hematoma density, and architecture classification and measurement techniques for radiological parameters also contributed to data heterogeneity. Evaluation of study quality using the NOS questionnaire revealed that the majority of studies did not reach maximum quality scores, mainly because no adjustments were performed for confounding factors and incomplete follow up information. However, the findings were generally consistent and in line with acknowledged clinically relevant parameters.

For the present study, we included only surgically treated CSDH patients by burr hole or twist drill craniostomy with subdural drainage, the mainstay treatment worldwide, in order to eliminate the potential effect of different treatment strategies on recurrence rates. We maintained a study protocol with strict inclusion and exclusion criteria in order to achieve good quality and homogeneous data as good as possible, which to our knowledge has provided the first data review on this subject.

\section{Conclusion}

From the present meta-analysis, we have derived several CT predictors that are associated with recurrence after surgical treatment of CSDH. In particular, CSDH with hyperdense components or with laminar or separated architecture type entail higher recurrence rates. Preoperative assessment of these parameters identifies a population with higher $\mathrm{CSDH}$ recurrence risk, and appreciation of these findings allows clinicians to apply an individualized management strategy. Future research is necessary to validate the prognostic value of these CT parameters in prospective studies and in particular investigate the value of a simplified density classification. Clear definitions and description of radiological measurement techniques are mandatory for a reliable evaluation.

Acknowledgements We want to thank the research bureau (Landsteiner Institute) of the Haaglanden Medical Center (HMC), The Jacobus Foundation (Stichting Jacobus), The Hague, information specialist J. W. Schoones, and the department of Epidemiology of the Leiden University Medical Center (LUMC) for their facilitation during this research.

Data Availability This systematic review and meta-analysis used already published data obtained from the literature search to conduct metaanalyses.

Funding No funding is received for this study.

\section{Compliance with ethical standards}

Conflicts of interest The authors declare that they have no conflict of interest.

Ethics approval For this type of study (systematic review and metaanalysis of current literature), formal consent is not required.

Consent to participate For this type of study (systematic review and meta-analysis of current literature), formal consent is not required.

Consent for publication For this type of study (systematic review and meta-analysis of current literature), formal consent for publication is not required.

Code availability For this type of study (systematic review and metaanalysis of current literature), code availability is not required.

Open Access This article is licensed under a Creative Commons Attribution 4.0 International License, which permits use, sharing, adaptation, distribution and reproduction in any medium or format, as long as you give appropriate credit to the original author(s) and the source, provide a link to the Creative Commons licence, and indicate if changes were made. The images or other third party material in this article are included in the article's Creative Commons licence, unless indicated otherwise in a credit line to the material. If material is not included in the article's Creative Commons licence and your intended use is not permitted by statutory regulation or exceeds the permitted use, you will need to obtain permission directly from the copyright holder. To view a copy of this licence, visit http://creativecommons.org/licenses/by/4.0/.

\section{References}

1. Almenawer SA, Farrokhyar F, Hong C, Alhazzani W, Manoranjan B, Yarascavitch B, Arjmand P, Baronia B, Reddy K, Murty N, Singh S (2014) Chronic subdural hematoma management: a systematic review and meta-analysis of 34,829 patients. Ann Surg 259:449-457

2. Kudo H, Kuwamura K, Izawa I, Sawa H, Tamaki N (1992) Chronic subdural hematoma in elderly people: present status on Awaji Island and epidemiological prospect. Neurol Med Chir 32:207-209 
3. Trotter W (1914) Chronic subdural hemorrhage of traumatic origin and its relation to pachymeningitis haemorhhagica interna. $\mathrm{Br} \mathrm{J}$ Surg 2:271-291

4. Drapkin AJ (1991) Chronic subdural hematoma: pathophysiological basis for treatment. Br J Neurosurg 5:467-473

5. Bosche B, Molcanyi M, Noll T, Kochanek M, Kraus B, Rieger B, el Majdoub F, Dohmen C, Löhr M, Goldbrunner R, Brinker G (2013) Occurrence and recurrence of spontaneous chronic subdural haematoma is associated with factor XIII deficiency. Clin Neurol Neurosurg 115:13-18

6. Shim YS, Park CO, Hyun DK, Park HC, Yoon SH (2007) What are the causative factors for a slow, progressive enlargement of a chronic subdural hematoma. Yonsei Med J 48:210-217

7. Ito H, Komai T, Yamamoto S (1978) Fibrinolytic enzyme in the lining walls of chronic subdural hematoma. J Neurosurg 48:197-200

8. Labadie EL, Glover D (1975) Local alterations of hemostaticfibrinolytic mechanisms in reforming subdural hematomas. Neurology 25:669-675

9. Bartek J, Sjåvik K, Kristiansson H, Stahl F, Fornebo I, Förander P et al (2017) Predictors of recurrence and complications after chronic subdural hematoma surgery: a population-based study. World Neurosurg 106:609-614

10. Liu W, Bakker NA, Groen RJ (2014) Chronic subdural hematoma: a systematic review and meta-analysis of surgical procedures. $\mathrm{J}$ Neurosurg 121:665-673

11. Sun TF, Boet R, Poon WS (2005) Non-surgical primary treatment of chronic subdural haematoma: preliminary results of using dexamethasone. Br J Neurosurg 19:327-333

12. Soleman JN, Mariani L (2017) The conservative and pharmacological management of chronic subdural haematoma. Swiss Med Wkly 147:w14398

13. Delgado-Lopez PD, Martin-Velasco V, Castilla-Diez JM, Rodriquez-Salazar A, Galacho-Harriero AM, FernandexArconada O (2009) Dexamethasone treatment in chronic subdural haematoma. Neurocirugia 20:346-359

14. Miah IP, Herklots M, Roks G, Peul WC, Walchenbach R, Dammers R, Lingsma HF, den HM H, Jellema K, van der NA G (2019) Dexamethasone Therapy in Symptomatic Chronic Subdural Hematoma (DECSA-R): a retrospective evaluation of initial corticosteroid therapy versus primary surgery. J Neurotrauma 37:366-372

15. Weigel R, Schmiedek P, Krauss JK (2003) Outcome of contemporary surgery for chronic subdural haematoma: evidence based review. J Neurol Neurosurg Psychiatry 74:937-943

16. Santarius T, Hutchinson PJ (2004) Chronic subdural haematoma: time to rationalize treatment? Br J Neurosurg 18:328-332

17. Santarius T, Kirkpatrick PJ, Ganesan D, Chia HL, Jalloh I, Smielewski P, Richards HK, Marcus H, Parker RA, Price SJ, Kirollos RW, Pickard JD, Hutchinson PJ (2009) Use of drains versus no drains after burr-hole evacuation of chronic subdural haematoma: a randomised controlled trial. Lancet 374:1067-1073

18. Matsumoto HH, Okada T, Sakurai Y, Minami H, Masuda A, Tominaga $S$ et al (2017) Clinical investigation of refractory chronic subdural hematoma: a comparison of clinical factors between single and repeated recurrences. World Neurosurg 107:706-715

19. Han MH, Ryu JI, Kim CH, Kim JM, Cheong JH, Yi HJ (2017) Predictive factors for recurrence and clinical outcomes in patients with chronic subdural hematoma. J Neurosurg 127:1117-1125

20. Amirjamshidi AA, Eftekhar B, Rashidi A, Rezaii J, Esfandiari K, Shirani A et al (2007) Outcomes and recurrence rates in chronic subdural haematoma. Br J Neurosurg 21:272-275

21. Shen J, Yuan L, Ge R, Wang Q, Zhou W, Jiang XC, Shao X (2019) Clinical and radiological factors predicting recurrence of chronic subdural hematoma: a retrospective cohort study. Injury 50:1634-1640
22. Altaf IS, Vohra AH (2018) Radiolological predictors of recurrence of chronic subdural hematoma. Pak J Med Sci 34:194-197

23. Chon KH, Lee JM, Koh EJ, Choi HY (2012) Independent predictors for recurrence of chronic subdural hematoma. Acta Neurochir 154:1541-1548

24. Ko BSL, Seo BR, Moon SJ, Kim JH, Kim SH (2008) Clinical analysis of risk factors related to recurrent chronic subdural hematoma. J Korean Neurosurg Soc 43:11-15

25. Jung Y, Jung N, El K (2015) Independent predictors for recurrence of chronic subdural hematoma. J Korean Neurosurg Soc $57: 266-270$

26. Nakaguchi HT, Yoshimasu N (2001) Factors in the natural history of chronic subdural hematomas that influence their postoperative recurrence. J Neurosurg 95:256-262

27. You W, Zhu Y, Wang Y, Liu W, Wang H, Wen L, Yang X (2018) Prevalence of and risk factors for recurrence of chronic subdural hematoma. Acta Neurochir 160:893-899

28. Yan CY, Huang JW (2018) A reliable nomogram model to predict the recurrence of chronic subdural hematoma after burr hole surgery. World Neurosurgery 118:e356-e366

29. Song DHK, Chun HJ, Yi HJ, Bak KH, Ko Y, Oh SJ (2014) The predicting factors for recurrence of chronic subdural hematoma treated with burr hole and drainage. Korean $\mathrm{J}$ Neurotrauma 10:41-48

30. Huang YHL, Lu CH, Chen WF (2014) Volume of chronic subdural haematoma: is it one of the radiographic factors related to recurrence? Injury 45:327-331

31. Huang YHY, Lee TC, Liao CC (2013) Bilateral chronic subdural hematoma: what is the clinical significance? Int J Surg 11:544-548

32. Jeong SIK, Won YS, Kwon YJ, Choi CS (2014) Clinical analysis of risk factors for recurrence in patients with chronic subdural hematoma undergoing burr hole trephination. Korean $\mathrm{J}$ Neurotrauma 10:15-21

33. Tugcu B, Tanriverdi O, Baydin S, Hergunsel B, Gunaldi O, Ofluoglu E et al (2014) Can recurrence of chronic subdural hematoma be predicted? A retrospective analysis of 292 cases. J Neurol Surg A Cent Eur Neurosurg 75:37-41

34. Jang KM, Chou HH, Mun HY, Nam TK, Park YS, Kwon JT (2020) Critical depressed brain volume influences the recurrence of chronic subdural hematoma after surgical evaluation. Nat Res Forum 10:1-8

35. Stang A (2010) Critical evaluation of the Newcastle-Ottawa scale for the assessment of the quality of nonrandomized studies in metaanalyses. Eur J Epidemiol 25:603-605

36. Won SY, Dubinski D, Eibach M, Gessler F, Herrmann E, Keil F et al (2020) External validation and modification of the Oslo grading system for predction of postoperative recurrence of chronic subdural hematoma. Neurosurg Rev:1-10. https://doi.org/10. 1007/s10143-020-01271-w

37. Spallone AG, Gagliardi FM, Vagnozzi R (1989) Chronic subdural hematoma in extremely aged patients. Eur Neurol 29:18-22

38. Tokmak MI, Bek S, Gokduman CA, Erdal M (2007) The role of exudation in chronic subdural hematomas. J Neurosurg 107:290-295

39. Weigel RH, Schilling L (2014) Vascular endothelial growth factor concentration in chronic subdural hematoma fluid is related to computed tomography appearance and exudation rate. J Neurotrauma $31: 670-673$

40. Fukuhara TG, Asari S, Ohmoto T, Akioka T (1996) The relationship between brain surface elastance and brain reexpansion after evacuation of chronic subdural hematoma. Surg Neurol 45:570-574

41. Sklar FH, Beyer CW, Clark WK (1980) Physiological features of the pressure-volume function of brain elasticity in man. J Neurosurg $53: 166-172$ 
42. D'Abbondanza JA, Macdonald RL (2014) Experimental models of chronic subdural hematoma. Neurol Res 36:176-188

43. Hammer AT, Kerry G, Schrey M, Hammer C, Steiner HH (2017) Predictors for recurrence of chronic subdural hematoma. Turk Neurosurg 27:756-762

44. Lee SHC, Lim DJ, Ha SK, Kim SD, Kim SH (2018) The potential of diffusion-weighted magnetic resonance imaging for predicting the outcomes of chronic subdural hematomas. J Korean Neurosurg Soc 61:97-104

45. Kim SUL, Kim YI, Yang SH, Sung JH, Cho CB (2017) Predictive factors for recurrence after burr-hole craniostomy of chronic subdural hematoma. J Korean Neurosurg Soc 60:701-709

46. Stavrinou P, Katsigiannis S, Lee JH, Hamisch C, Krischek B, Mpotsaris A, Timmer M, Goldbrunner R (2017) Risk factors for chronic subdural hematoma recurrence identified using quantitative computed tomography analysis of hematoma volume and density. World Neurosurg 99:465-470

47. Goto HI, Nomura M, Tanaka K, Nomura S, Maeda K (2015) Magnetic resonance imaging findings predict the recurrence of chronic subdural hematoma. Neurol Med Chir 55:173-178

48. Stanisic M, Hald J, Rasmussen IA, Pripp AH, Ivanovic J, Kolstad F et al (2013) Volume and densities of chronic subdural haematoma obtained from CT imaging as predictor of postoperative recurrence: a prospective study of 107 operated patients. Acta Neurochir 155:323-333

49. Yamamoto HH, Hamada H, Hayashi N, Origasa H, Endo S (2003) Independent predictors of recurrence of chronic subdural hematoma: results of multivariate analysis performed using a logistic regression model. J Neurosurg 98:1217-1221

50. Oishi MT, Tamatani S, Kitazawa T, Saito M (2001) Clinical factors of recurrent chronic subdural hematoma. Neurol Med Chir 41:382-386

51. Santarius TQ, Sivakumaran R, Kirkpatrick PJ, Kirollos RW, Hutchinson PJ (2010) The role of external drains and peritoneal conduits in the treatment of recurrent chronic subdural hematoma. World Neurosurg 73:747-750

52. Lee KSB, Bae HG, Doh JW, Yun IG (1997) The computed tomographic attenuation and the age of subdural hematomas. J Korean Med Sci 12:353-359

53. Sieswerda-Hoogendoorn T, Postema FAM, Verbaan D, Majoie CB, van RR R (2014) Age determination of subdural hematomas with CT and MRI: a systematic review. Eur J Radiol 83:1257-1268

54. Bergstrom ME, Levander B, Svendsen P (1977) Computed tomography of cranial subdural and epidural hematomas: variation of attenuation related to time and clinical events such as rebleeding. J Comput Assist Tomogr 1:449-455

55. Scotti GT, Melancon D, Belanger G (1977) Evaluation of the age of subdural hematomas by computerized tomography. J Neurosurg 47:311-315

56. Fujisawa HN, Tsuchida E, Ito H (1998) Serum protein exudation in chronic subdural haematomas: a mechanism for haematoma enlargement? Acta Neurochir 140:161-165

57. Gorelick PB, Weisman SM (2005) Risk of hemorrhagic stroke with aspirin use: an update. Stroke 36:1801-1807

58. Frati AS, Mainiero F, Ippoliti F, Rocchi G, Raco A, Caroli E et al (2004) Inflammation markers and risk factors for recurrence in 35 patients with a posttraumatic chronic subdural hematoma: A prospective study. J Neurosurg 100:24-32

59. Nomura SK, Fujisawa H, Ito H, Nakamura K (1994) Characterization of local hyperfibrinolysis in chronic subdural hematomas by SDSPAGE and immunoblot. J Neurosurg 81:910-913

60. Kitazono MY, Satoh H, Onda H, Matsumoto G, Fuse A, Teramoto A (2012) Measurement of inflammatory cytokines and thrombomodulin in chronic subdural hematoma. Neurol Med Chir $52: 810-815$

61. Nakamura ST (1989) Extraction of angiogenesis factor from chronic subdural haematomas. Significance in capsule formation and haematoma growth. Brain Inj 3:129-136

62. Bosche B, Molcanyl M, Rej S, Doeppner TR, Obermann M, Müller DJ et al (2016) Low-dose lithium stabilizes human endothelial barrier by decreasing MLC phosphorylation and universally augments cholinergic vasorelaxation capacity in a direct manner. Front Physiol 7:1-12

63. Bosche B, Schäffer M, Graf R, Härtel FV, Schäfer U, Noll T (2013) Lithium prevents early cytosolic calcium increase and secondary injurious calcium overload in glycolytically endothelial cells. Biochem Biophys Res Commun 434:268-272

64. Ohba SK, Nakagawa T, Murakami H (2013) The risk factors for recurrence of chronic subdural hematoma. Neurosurg Rev 36:145-149

65. Nayil KR, Sajad A, Zahoor S, Wani A, Nizami F, Laharwal M (2012) Subdural hematomas: an analysis of 1181 Kashmiri patients. World Neurosurg 77:103-110

66. Desai VRS, Britz GW (2017) Management of recurrent subdural hematomas. Neurosurg Clin N Am 28:279-286

67. Gelabert-Gonzalez MIP, Garcia-Allut A, Martinez-Rumbo R (2005) Chronic subdural haematoma: surgical treatment and outcome in 1000 cases. Clin Neurol Neurosurg 107:223-229

68. Tanikawa MM, Yamada K, Yamashita N, Matsumoto T, Banno T, Miyati T (2001) Surgical treatment of chronic subdural hematoma based on intrahematomal membrane structure on MRI. Acta Neurochir 143:613-618

69. El-Kadi HM, Kaufman HH (2000) Prognosis of chronic subdural hematomas. Neurosurg Clin N Am 11:553-567

70. Kang MSK, Kwon HJ, Cho SW, Kim SH, Youm JY (2007) Factors influencing recurrent chronic subdural hematoma after surgery. J Korean Neurosurg Soc 41:11-15

71. Mori KM (2001) Surgical treatment of chronic subdural hematoma in 500 consecutive cases: clinical characteristics, surgical outcome, complications, and recurrence rate. Neurol Med Chir 41:371-381

72. Torihashi K, Sadamasa N, Yoshida K, Narumi O, Chin M, Yamagata S (2008) Independent predictors for recurrence of chronic subdural hematoma: a review of 343 consecutive surgical cases. Neurosurgery 63:1125-1129

73. Qian ZY, Sun F, Sun Z (2017) Risk factors for recurrence of chronic subdural hematoma after burr hole surgery: potential protective role of dexamethasone. Br J Neurosurg 31:84-88

74. Motoie RK, Otsuji R, Ren N, Nagaoka S, Maeda K, Ikai Y et al (2018) Recurrence in 787 Patients with chronic subdural hematoma: retrospective cohort investigation of associated factors including direct oral anticoagulant use. World Neurosurg 118:e87-e91

75. Motiei-Langroudi RS, Shi S, Adeeb N, Gupta R, Griessenauer CJ, Papavassiliou E et al (2018) Factors predicting reoperation of chronic subdural hematoma following primary surgical evacuation. J Neurosurg 129:1143-1150

76. Stanisic MLJ, Mahesparan R (2005) Treatment of chronic subdural hematoma by burr-hole craniostomy in adults: influence of some factors on postoperative recurrence. Acta Neurochir 147:1249-1256

77. Adachi A, Higuchi Y, Fujikawa A, Machida T, Sueyoshi S, Harigaya K, Ono J, Saeki N (2014) Risk factors in chronic subdural hematoma: comparison of irrigation with artificial cerebrospinal fluid and normal saline in a cohort analysis. PLoS One 9:e103703

Publisher's note Springer Nature remains neutral with regard to jurisdictional claims in published maps and institutional affiliations. 


\section{Affiliations}

Ishita P. Miah ${ }^{1}$ (D) $\cdot$ Yeliz Tank $^{2} \cdot$ Frits R. Rosendaal $^{3} \cdot$ Wilco C. Peul $^{1,4} \cdot$ Ruben Dammers $^{5} \cdot$ Hester F. Lingsma $^{6}$. Heleen M. den Hertog ${ }^{7} \cdot$ Korné Jellema ${ }^{4}$. Niels A. van der Gaag ${ }^{4,8} \cdot$ on behalf of the Dutch Chronic Subdural Hematoma Research Group

1 Department of Neurology and Neurosurgery, Leiden University Medical Center (LUMC), Albinusdreef 2, 2333, ZA

Leiden, The Netherlands

2 Department of Radiology, Leiden University Medical Center (LUMC), Albinusdreef 2, 2333, ZA Leiden, The Netherlands

3 Department of Clinical Epidemiology, Leiden University Medical Center (LUMC), Albinusdreef 2, 2333, ZA Leiden, The Netherlands

4 Department of Neurology and Neurosurgery, Haaglanden Medical Center (HMC), Lijnbaan 32, 2512, VA The Hague, The Netherlands
5 Department of Neurosurgery, Erasmus Medical Center (EMC), Erasmus MC Stroke Center, Dr. Molewaterplein 40, 3015, GD Rotterdam, The Netherlands

6 Department of Public Health and Medical Decision Making, Erasmus Medical Center (EMC), Dr. Molewaterplein 40, 3015, GD Rotterdam, The Netherlands

7 Department of Neurology, Isala Hospital Zwolle, Dokter van Heesweg 2, 8025, AB Zwolle, The Netherlands

8 Department of Neurosurgery, Haga Teaching Hospital, Els BorstEilersplein 275, 2545, AA The Hague, The Netherlands 